\title{
The Frequency of Fibromyalgia Syndrome and the Quality of Life in Patients with Peritoneal Dialysis
}

\author{
Muyesser Okumus*, Hulya Parpucu, Seher Kocaoglu, Esma Ceceli, Murat Duranay, Punar Borman
}

The Ministry of Health Ankara Training and Research Hospital, Clinics of Physical Medicine and Rehabilitation and Clinics of Nephrology, Ankara, Turkey.

Email: *drmuyesser@hotmail.com

Received June $16^{\text {th }}, 2012$; revised July $22^{\text {nd }}, 2012$; accepted August $7^{\text {th }}, 2012$

\begin{abstract}
Objective: The aim of this study was to determine the frequency of fibromyalgia (FM) in patients on Peritoneal Dialysis (PD) and to investigate its impact on the quality of life of that population. Methods: A hundred and twenty four patients with end stage renal disease who had undergone PD, and a control group of 54 age and sex-matched healthy volunteers were included to the study. Demographic characteristics including age, sex, disease and dialysis duration were recorded. Fibromyalgia Impact Questionnaire (FIQ) and Nottingham Health Profile (NHP) were also measured. Results: The mean age of the patients ( 68 female, 56 male) and control subjects ( 36 female, 18 male) were $43.5 \pm 13.4$ and $41.2 \pm 9.2$ years respectively. The prevalence of FM in the PD patients and controls were determined as $9.7 \%$ (12 patients) and $11.1 \%$ (6 controls) respectively and were found to be similar $(\mathrm{p}=0.983)$. The mean score of subgroups of NHP except pain and emotion subgroups, were significantly higher in PD patients than in the control group. The mean FIQ levels, the scores of all the subgroups of NHP except the social subgroup were significantly higher in PD patients with FM than in those without FM. Conclusion: In conclusion although the prevalence of FM appears to be similar in PD patients and control subjects, the functional disability is common and quality of life is worse in PD patients with FM than in patients without FM. Detection and treatment of FM may lead to improvement in the quality of life of PD patients with this syndrome.
\end{abstract}

Keywords: Fibromyalgia; Dialysis; Chronic Kidney Disease; Quality of Life

\section{Introduction}

Fibromyalgia (FM) is characterized by chronic widespread pain, fatigue, and sleep disturbances. American College of Rheumatology 1990 Fibromyalgia Classification Criteria is based on a history of chronic widespread musculoskeletal pain for at least 3 months and more than 11 painful tender points with palpation [1]. FM is frequently accompanied by fatigue, anxiety, sleep disturbance, headache and irrittable bowel syndrome [2]. The etiology of FM is unknown and its pathogenesis is not clearly understood. It is a more common entity among women. The prevalence of FM among the woman population is $3.5 \%$ in the USA and $3.6 \%$ in Turkey $[3,4]$. The effects of FM on quality of life are well known in the literature [5]. Because musculoskeletal pain is regarded as the most prevalent form of chronic pain in Chronic Kidney Disease (CKD), a differential diagnosis of FM should be considered in that group of patients [6]. Moreover, hemodialysis (HD) patients may develop severe forms of secondary hyperparathyroidism (SHPT), a pain-

${ }^{*}$ Corresponding author. ful syndrome of which symptoms may overlap with those of FM [1]. There were 2 studies on FMS in the HD population $[7,8]$ but no study was performed about the presence of FM patients with Peritoneal Dialysis (PD).

The aim of this study was to determine the the frequency of FM in patients on PD, and to investigate its impact on the quality of life in patients suffering from this chronic condition. The relationship between disease characteristics, functional status and quality of life were also determined in all subjects.

\section{Material and Methods}

A hundred and twenty four patients with end stage renal disease who had undergone PD 4 or 5 times daily from outpatients clinics of nephrology were referred to Rheumatology unit of Physical Medicine and Rehabilitation Clinic, between March and June 2010. Fifty four age similar control subjects who were admitted to outpatients clinics of Physical Medicine and Rehabilitation Clinic for musculoskeletal pain, and who had volunteered to participate in the study, were included. The inclusion criteria 
for FM group were generalized pain both in the upper and lower extremities and both in the left and right sides of the body. Patients having unregulated diabetic disease, thyroid disorders, chronic rheumatic diseases and neuropathic diseases were excluded from the study. FM was diagnosed according to the ACR classification criteria detailed above [1]. Subjects were asked if they had experienced chronic widespread pain for at least 3 months. Each subject was also examined for tenderness at 18 tender points by digital palpation. One score point was assigned for each tender point noted; thus, each individual's tender point score was between 0 and 18. Demographic characteristics including age, sex, body mss index (BMI), disease, dialysis duration and the cause of Chronic Kidney Disease (CKD) were recorded. Associated symptoms comprising fatigue, morning stiffness, headace, irritable bowel syndrome, sleep disturbance, depression, anxiety, Raynaud phenomen, restless leg syndrome, paraesthesia histories of patients were also evaluated [9]. For each PD patient, the aetiology of renal failure and duration of PD were recorded. As the diagnosis of FM was evaluated in the current study, no patients were using specific medications for FM, such as opioid analgesics, codein or anti-depressants. Some patients reported sporadic use of common analgesics.

Fibromyalgia Impact Questionnaire (FIQ) [10] was used to measure the components of health status of the PD patients and controls. FIQ is a self-administered instrument and composed of 10 items. The first item related to patient's ability to perform daily tasks (cooking, cleaning, walking, driving, etc.). Items 2 and 3 assess the number of days they felt well in the past week and the number of days they were unable to work. The next seven items focus on the ability of patients to work, pain, fatigue, tiredness, stiffness, anxiety and depression. The Turkish version of the instrument was shown to be valid and reliable [11]. The quality of life of the all subjects was assessed by Nottingham Health Profile (NHP) [12]. In NHP, there are six categories in 38 questions consisting of energy, pain, physical mobility, sleep, emotional reactions and social isolation. The adaptation study of the Turkish version was also done, and the questionnaire was reported to be valid and reliable [13].

For the PD patients, the results of laboratory tests were recorded. Serum calcium, phosphorus, alkaline phosphatase, parathyroid hormone, urea, creatinine, sodium, potassium, uric acid, $\beta$ microglobulin, C-Reactive Protein (CRP), Erythrocyte Sedimentation Rate (ESR) were determined and the dialysis adequacy (KT/v) was calculated.

We obtained informed consent from all the patients and controls, and the study was approved by the ethics committee of the hospital.

Descriptive statistics were performed and indicated as mean + standard deviation and median (maximum-minimum) for continuous variables. All qualitative data are expressed as frequencies and percentages. The normality of the distribution of continuous variables was analyzed by Shapiro Wilk test. The intergroup comparisons for continuous variables between the two groups (patients and control) were performed by student $t$ test and Mann Whitney $U$ test. Chi square tests were used to define the categorical variables. The level of significance was set to 0.05. All statistical analyses were done using SPSS for windows version 15.0 program.

\section{Results}

A hundred and twenty four patients and 54 controls with the mean age of $43.5 \pm 13.4$ and $41.2 \pm 9.2$ years respectively, were included to the study. The mean duration of PD was $34.3 \pm 29.8$ months. The demographic and clinical characteristics of the PD patients and control group were shown in Table 1 The prevalence of FM in the PD patients and controls were 9.7\% (12 patients) and $11.1 \%$ (6 controls) respectively. The frequency of FM was found to be similar between the groups $(p=0.983)$. The mean age of the 18 (PD patients with FM and controls with FM; aged $40.1 \pm 4.4$ years) participants with FM was significantly different from that of the remaining participants (aged $43.1 \pm 12.9$ years) $(p=0.042)$, and also there was statistical difference between the mean age of PD patients with FM (aged $41.7 \pm 4.9$ years) and the mean age of the controls with FM (aged $38.0 \pm 2.4$ years) $(p=0.038)$. The FM prevalence in women and men were similar in PD patients but all of the subjects with FM (aged $38.0 \pm 2.4$ years) in the control group were women.

The duration of PD $(28.0 \pm 12.4$ months $)$, BMI $(24.6 \pm$ 4.7) and age $(41.2 \pm 4.9)$ in the PD patients with FM were not statistically different from the PD patients without FM (35.5 \pm 32.0 months, $24.3 \pm 4.6$ and $43.8 \pm$ 14.1 respectively) $(\mathrm{p}>0.05)$.

The mean score of subgroups of NHP except pain and emotion subgroups were significantly higher in PD patients than in the control group (Table 2). The mean FIQ levels, except the social subgroup of NHP, the score of all the subgroups of NHP and the number of FM associated symptoms were significantly higher than in PD patients with FM than in those without FM (Table 3).

Within the PD patients laboratory parameters were not different between the patients with FM and without FM $(p>0.05)$ (Table 4). FM associated symptoms such as fatigue, morning stiffness, headache and restless leg syndrome were significantly more common in the PD patients with FM than in the PD patients without FM (Table 5).

\section{Discussion}

Our study is the first study in the literature that evaluates 
Table 1. The demographic and clinical characteristics of the PD patients $(n=124)$.

\begin{tabular}{ccc}
\hline & $\begin{array}{c}\text { Peritoneal dialysis group } \\
(\mathrm{n}=124)\end{array}$ & $\begin{array}{c}\text { Control group } \\
(\mathrm{n}=54)\end{array}$ \\
\hline Age (years) & $43.5 \pm 13.4$ & $41.2 \pm 9.2$ \\
Sex (female/male) $\mathrm{n}(\%)$ & $68 / 56(54.8 / 45.2)$ & $36 / 18(66.7 / 33.3)$ \\
BMI $\left(\mathrm{kg} / \mathrm{m}^{2}\right)$ & $24.6 \pm 4.7$ & $25.9 \pm 3.9$ \\
Cause of CKD n (\%) & - & - \\
Unknown & $42(33.8)$ & - \\
Hypertension & $26(21)$ & - \\
Diabetes mellitus & $18(14.5)$ & - \\
Pyelonephritis & $10(8.1)$ & - \\
Glomerulonephritis & $6(4.8)$ & - \\
Amyloidosis due to FMF & $8(6.5)$ & \\
Other & $14(11.3)$ & \\
\hline
\end{tabular}

PD: Peritoneal Dialysis, CKD: Chronic Kidney Disease.

Table 2. The comparison of FIQ, NHP questionnaires and number of FM associated symptoms of patients with PD and control group.

\begin{tabular}{cccc}
\hline & PD group & Control group \\
\hline FIQ & $33.0 \pm 24.6$ & $27.9 \pm 17.5$ \\
NHP-pain & $18.1 \pm 22.9$ & $21.2 \pm 26.5$ & 0.119 \\
NHP-function & $19.6 \pm 22.2$ & $9.7 \pm 13.8$ & 0.432 \\
NHP-fatigue & $29.8 \pm 40.8$ & $16.7 \pm 23.8$ \\
NHP-sleep & $28.1 \pm 31.1$ & $15.6 \pm 24.8$ \\
NHP-social & $15.2 \pm 25.7$ & $2.2 \pm 6.3$ \\
NHP-emotion & $28.0 \pm 30.8$ & $26.0 \pm 29.3$ \\
\# of FM associated symptoms & $3.1 \pm 2.2$ & $3.5 \pm 2.0$ \\
\hline
\end{tabular}

PD: Peritoneal Dialysis, FM: fibromyalgia syndrome, FIQ: Fibromyalgia Impact Questionnaire, NHP: Nottingham Health Profile.

Table 3. The comparison of FIQ, NHP questionnaires and number of FM associated symptoms of PD patients with FM and those without FM.

\begin{tabular}{cccc}
\hline & PD with FM & PD without FM & p \\
\hline FIQ & $68.3 \pm 8.4$ & $291 \pm 22.6$ & 0.001 \\
NHP-pain & $56.5 \pm 22.4$ & $14.0 \pm 19.0$ & 0.001 \\
NHP-function & $35.4 \pm 19.0$ & $17.9 \pm 22.0$ & 0.003 \\
NHP-fatigue & $58.3 \pm 35.9$ & $26.8 \pm 40.2$ & 0.004 \\
NHP-sleep & $66.7 \pm 23.1$ & $24.0 \pm 29.0$ & 0.001 \\
NHP-social & $23.3 \pm 37.0$ & $14.3 \pm 24.2$ & 0.245 \\
NHP-emotion & $68.8 \pm 16.4$ & $23.5 \pm 28.7$ & 0.001 \\
\# of FM associated symptoms & $5.7 \pm 2.0$ & $2.8 \pm 2.1$ & 0.001 \\
\hline
\end{tabular}

PD: Peritoneal Dialysis, FM: fibromyalgia syndrome, FIQ: Fibromyalgia Impact Questionnaire, NHP: Nottingham Health Profile. 
Table 4. Laboratory parameters in the PD patients with FM and those without FM.

\begin{tabular}{cccc}
\hline & PD with FM & PD without FM & p \\
\hline Alkaline phosphatase U/L & $142.2 \pm 45.4$ & $141.7 \pm 169.8$ & 0.119 \\
Creatinine mg/dL & $8.7 \pm 3.6$ & $9.0 \pm 2.7$ & 0.636 \\
Urea mg/dL & $116.3 \pm 32.9$ & $103.6 \pm 27.3$ & 0.460 \\
PTH pg/mL & $504.8 \pm 321.1$ & $584.3 \pm 484.5$ & 0.850 \\
Calcium mg/dL & $8.7 \pm 0.7$ & $8.9 \pm 0.7$ & 0.519 \\
Phosphorus mg/dL & $5.3 \pm 1.9$ & $4.6 \pm 1.0$ & 0.531 \\
Uric acid mg/dL & $5.4 \pm 0.7$ & $5.4 \pm 1.2$ & 0.955 \\
$\beta$ microglobulin mg/dL & $24.3 \pm 7.2$ & $25.4 \pm 6.1$ & 0.980 \\
Vitamin B 12 pg/mL & $340.5 \pm 118.7$ & $378.8 \pm 182.9$ & 0.719 \\
Ferritin ng/mL & $431.2 \pm 245.2$ & $488.2 \pm 281.4$ & 0.649 \\
ESR mm/hr & $27.7 \pm 21.4$ & $40.1 \pm 26.3$ & 0.304 \\
C-reactive protein mg/dL & $0.8 \pm 0.6$ & $2.4 \pm 4.0$ & 1.000
\end{tabular}

$\mathrm{Kt} / \mathrm{V}$. İndex of dialysis adequacy, PTH: Parathyroid hormone, PD: Peritoneal Dialysis, FM: fibromyalgia syndrome.

Table 5. FM related clinical features of the PD patients with FM and without FM.

\begin{tabular}{cccc}
\hline & PD patients with FM & PD patients without FM & P \\
\hline Fatigue (\%) & 100 & 58.9 & 0.004 \\
Morning stiffness (\%) & 50 & 14.3 & 0.007 \\
Headache (\%) & 83.3 & 46.4 & 0.033 \\
Irritable bowel syndrome (\%) & 16.7 & 7.1 & 0.906 \\
Sleep disturbance (\%) & 50 & 48.2 & 0.026 \\
Depression (\%) & 66.7 & 32.1 & 1.000 \\
Anxiety (\%) & 50 & 50 & 0.046 \\
Raynaud phenomen (\%) & 16.7 & 1.8 & 0.000 \\
Restless leg syndrome (\%) & 83.3 & 17.9 & 0.002 \\
Paraesthesia (\%) & 50 & 10.7 & \\
\hline
\end{tabular}

PD: Peritoneal Dialysis, FM: fibromyalgia syndrome.

FM and its impact on the quality of life of PD patients. Rheumatic disorders are frequent complications of renal disease, and most data indicate that the risk of such complications may increase with time on PD. To date, no reports have published the prevalence rates of FM in PD patients. The prevalence of FM is ranged from $0.5 \%$ to $6 \%$ and females are more affected than men in the general population [3]. The prevalence rates of FM in PD patients and controls were $9.7 \%$ and $11.1 \%$ in our study. Half of PD patients with FM were men while all the control group with FM were female in our study in contrast with the previous studies [1].

The previous studies on HD patients found that the prevalence rates of FM were $3.9 \%$ and $7.4 \%$ in patients with HD respectively $[7,8]$. Similar to these studies we indicated no differences between the frequency rates of FM in PD patients and control subjects. In contrast with the above mentioned studies we found that the frequency of women and men with FM on PD was similar which may be due to the biased selection of our study population. Concerning age, it has been assumed that the prevalence of FM increases with age. Wolfe et al. showed 
that the highest rates of FM are seen in subjects in their $60 \mathrm{~s}$ and $70 \mathrm{~s}$ [3]. In our study, our FM patients seem to be younger, as $77.8 \%$ of them aged $39-50$ years. Similar to our results, $54 \%$ and $58 \%$ of FM patients with HD aged $31-50$ and $39-50$ years respectively $[7,8]$. Because the number of our subjects in different age groups was low, it is inconvenient to find the prevalence of FM according to different age groups. The mean age of the PD patients with FM was found to be significantly higher than the controls with FM in our study.

The incidence of musculoskeletal problems increases with time on dialysis [14]. In addition to this, malnutrition may also frequently affect the health status of the dialysis patients [15]. However we could not find any significant difference regarding duration of PD and BMI between the PD patients with FM and those without FM.

Despite there is no specific laboratory tests to diagnosis FM, we also compared the laboratory parameters between the PD patients with FM and those without FM. Analysis of the PD patient data conceived no significant differences between these groups. These results underlined the fact that laboratory parameters do not affect the status of FM.

Each of our subjects completed the FIQ. With in the PD group, the patients with FM had higher FIQ score than those without FM. These results indicate that FIQ questionnaire reflects general health status and factors that affect daily activity but FM causes additional functional disability and has distinct negative effects on general health, even within chronic states such as CKD [8].

The many features that frequently accompany FM include fatigue, sleep disturbance, irritable bowel, paraesthesia, psychological problems, restless leg syndrome [16-18]. We determined that the frequencies of headache, sleep disturbance, restless leg syndrome and paraesthesia were higher than in control subjects, regardless of whether or not the individual was diagnosed as having FM. This is not surprising, since musculoskeletal and psychological problems are common in patients on PD, and are related to metabolic and neurological disorders [19-22]. We also detected that, fatigue, morning stiffness, headache and restless leg syndrome symptoms were more frequent in the PD patients with FM than those without FM. But only the symptoms of restless leg syndrome were found to be more frequent in PD patients with FM than in controls with FM.

In patients with CKD, initiation of long term dialysis treatment increases survival but quality of life remains impaired compared with successful renal allo-transplantation $[23,24]$. In this study we also aimed to evaluate the quality of life in the PD patients and we compared it for patients with FM. Except social function, all of the NHP subgroups were significantly higher in PD patients with FM than those without FM. The scores of functional, fatigue, sleep disturbance and social subgroups of NHP were also significantly higher in PD patients than controls regardless of FM. This was not surprising, because musculoskeletal and psychological problems can be related to metabolic and neurological disorders [19]. In our study duration of PD and adequacy of PD (index of dialysis: $\mathrm{Kt} / \mathrm{V}$ ) were found to have no impact on PD patients with FM.

In conclusion although the prevalence of FMS appears to be similar in PD patients and control subjects, the frequencies of the associated symptoms are higher in PD patients. The functional disability is common and quality of life is worse in PD patients with FM than in patients without FM syndrome. Detection and treatment of FM may lead to improvement in the quality of life of PD patients with this syndrome. Therefore, we suggest the evaluation of the symptoms of FM in patients receiving PD.

\section{REFERENCES}

[1] F. Wolfe, H. A. Smyte, M. B. Yunus, R. M. Bennet, C. Bombardier, D. L. Goldenberg, P. Tugwell, S. M. Campell, M. Abeles, P. Clark, et al., "The American Collage of Rheumatology 1990 Criteria for Classification of Fibromyalgia: Report of the Multicentre Criteria Committee," Arthritis \& Rheumatism, Vol. 33, No. 2, 1990, pp. 160-172. doi:10.1002/art.1780330203

[2] S. Özkurt, A. Dönmez, M. Z. Karagülle, E. Uzunoğlu, M. Turan and N. Erdoğan, "Balneotherapy in Fibromyalgia: A Single Blind Randomized Controlled Clinical Study," Rheumatology International, Vol. 32, No. 7, 2012, pp. 19491954. doi:10.1007/s00296-011-1888-9

[3] F. Wolfe, K. Ross, J. Anderson, I. J. Russel and L. Hebert, "The Prevalence and Charecteristics of Fibromyalgia in the General Population," Arthritis \& Rheumatism, Vol. 38, No. 1, 1995, pp. 19-28. doi:10.1002/art.1780380104

[4] M. Topbaş, H. Çakırbay and H. Güleç, "The Prevalence of Fibromyalgia in Women Aged 20 - 64 in Turkey," Scandinavian Journal of Rheumatology, Vol. 34, No. 2, 2005, pp. 140-144.

[5] L. Neumann and D. Buskila, "Quality of Life and Physical Functioning of Relatives of Fibromyalgia Patients," Seminars in Arthritis and Rheumatism, Vol. 26, No. 6, 1997, pp. 834-839. doi:10.1016/S0049-0172(97)80027-3

[6] T. Bardin, "Musculoskeletal Manifestations of Chronic Renal Failure. Systemic Disorders with Rheumatic Manifestations," Current Opinion in Rheumatology, Vol. 15, No. 1, 2003, pp. 48-54. doi:10.1097/00002281-200301000-00009

[7] C. I. Couto, J. Natour and A. B. Carvalho, "Fibromyalgia: Its Prevalence and İmpact on the Quality of Life on a Hemodialyzed Population," Hemodialysis International, Vol. 12, No. 1, 2008, pp. 66-72. doi:10.1111/j.1542-4758.2008.00243.x

[8] T. E. Yuceturk, A. E. Yucel, H. Yuceturk, H. Kart-Koseoglu, R. Unuvar, F. N. Ozdemir and Z. Akcali, "Fibromyalgia: İts Prevalence in Hemodialysis Patients and İts Relationships with Clinical and Laboratory Parameters," Ne- 
phrology Dialysis Transplantation, Vol. 20, No. 11, 2005, pp. 2485-2488. doi:10.1093/ndt/gfi028

[9] W. G. Thompson, G. F. Longstreth, D. A. Drossman, K. W. Heaton, E. J. Irvine and S. A. Muller-Lissner, "Functional Bowel Disorders and Functional Abdominal Pain," Gut, Vol. 45, No. 2, 1999, pp. 1143-1147.

doi:10.1136/gut.45.2008.ii43

[10] C. S. Burckhardt, S. R. Clark and R. M. Bennett, "The Fibromyalgia İmpact Questionnaire: Development and Validation," The Journal of Rheumatology, Vol. 8, No. 5, 1991, pp. 728-733.

[11] S. Sarmer, S. Ergin and G. Yavuzer, "The Validity and Realibility of the Turkish Version of the Fibromyalgia İmpact Questionnaire," Rheumatology International, Vol. 20, No. 1, 2000, pp. 9-12. doi:10.1007/s002960000077

[12] T. D. Wade, "Measures of Handicap and Quality of Life," In: T. Wade, Ed., Measurement in Neurological Rehabilitation, Oxford University Press, London, 1992, pp. 231259.

[13] A. A. Kucukdeveci, S. P. Mckenna, S. Kutlay, Y. Gursel, D. Whalley and T. Arasil, "The Development and Psychometric Assessment of the Turkish Version of the Nottingham Health Profile," International Journal of Rehabilitation Research, Vol. 23, No. 1, 2000, pp. 31-38.

[14] A. E. Yucel, H. Kart-Koseoglu, I. Isklar, E. Kuruinci, F. N. Ozdemir and H. Arslan, "Bone Mineral Density in Patients on Maintenance Hemodialysis and Effect of Chronic Hepatitis C-Virus Enfection," Renal Failure, Vol. 26, No. 2, 2004, pp. 159-164. doi:10.1081/JDI-120038501

[15] M. P. Merkus, K. J. Jager, F. W. Dekker, R. J. de Haan, E. W. Boeschoten and R. T. Krediet, "Predictors of Poor Outcome in Chronic Dialysis Patients: The Netherlands Cooperative Study on the Adequacy of Dialysis. The NECOSAD Study Group," American Journal of Kidney Diseases, Vol. 35. No. 1, 2000, pp. 69-79. doi:10.1016/S0272-6386(00)70304-0

[16] S. Roizenbatt, H. Moldofsky, A. A. Benedito-Silva and S. Tufik, "Alpha Sleep Characteristic in Fibromyalgia," Arthritis and Rheumatism, Vol. 44, No. 1, 2001, pp. 222-230.

[17] G. Tridafilopoulus, R. W. Simms and D. L. Goldenderb, "Bowel Dysfunction in Fibromyalgia Syndrome," Diges- tive Diseases and Sciences, Vol. 36, No. 1, 1991, pp. 5964. doi:10.1007/BF01300088

[18] F. Wolfe, M. A. Cathey, S. M. Kleinheksel, S. P. Amos, R. G. Hoffman, D. Y. Young and D. J. Hawley, "Psychological Status in Primary Fibrositis and Fibrositis Associated with Rhematoid Arthritis," The Journal of Rheumatology, Vol. 11, No. 4, 1984, pp. 500-506.

[19] D. J. Sherrard, G. Herez, Y. Pei, N. A. Maloney, C. Greenwood, A. Manuel, C. Saiphoo, S. S. Fenton and G. V. Segre, "The Spectrum of Bone Disease in End-Stage Renal Failure-An Evolving Disorder," Kidney International, Vol. 43, No. 2, 1993, pp. 436-442. doi:10.1038/ki.1993.64

[20] D. Wendling and M. Guidet, "Articular and Periarticular Manifestations of Chronic Hemodialysis in the Adult," Revue du Rhumatisme et des Maladies Ostéo-Articulaires, Vol. 53, No. 12, 1986, pp. 715-721.

[21] K. C. Siamopoulos, M. Dardamanis, D. Kyriaki, M. Pappas, G. Siferopoulos and V. Alevisou, "Pituitary Adrenal Responsivenes to CRF in Chronic Uremic Patients," Peritoneal Dialysis International, Vol. 10, No. 2, 1990, pp. 153156.

[22] E. M. Kaptein, "Thyroid Hormone Metabolism and Thyroid Diseases in Chronic Renal Failure," Endocrine Reviews, Vol. 17, No. 1, 1996, pp. 45-63. doi:10.1210/edrv-17-1-45

[23] E. G. Lowrie, J. M. Lazarus, A. J. Mocelin, G. L. Bailey, C. L. Hampers, R. E. Wilson and J. P. Merrill, "Survival of Patients Undergoing Chronic Hemodialysis and Renal Transplantation," The New England Journal of Medicine, Vol. 288. No. 17, 1973, pp. 863-867. doi:10.1056/NEJM197304262881701

[24] R. W. Evans, D. L. Manninen, L. P. Garrison, L. G. Hart, C. R. Blagg, R. A. Gutman, A. R. Hull and E. G. Lowrie, "The Quality of Life Patients with End-Stage Renal Disease," The New England Journal of Medicine, Vol. 312, No. 9, 1985, pp. 553-559. doi:10.1056/NEJM198502283120905 